\title{
Toward Human-like Motion Planning in Urban Environments
}

\author{
Tianyu $\mathrm{Gu}^{1}$ and John M. Dolan ${ }^{2}$
}

\begin{abstract}
Prior autonomous navigation systems focused on the demonstration of the technological feasibility. But as the technology evolves, improving user experience through learning expert's or individual's driving pattern emerges as a promising research direction. As a first step toward this goal, we investigate methods to learn from human demonstrations in urban scenarios without any environmental disturbances (traffic-free). We propose a path model that generates a reference path with smooth and peak-value-reduced curvature, and a parameterized speed model to be fitted by human driving data. Model parameters are then learned through regression methods, and certain statistical human driving patterns are revealed. The learned model is then evaluated by comparing the generated plan with the collected data by the same human driver.
\end{abstract}

\section{INTRODUCTION}

The high cost of car crashes, the trillions of hours squandered in traffic jams, and the lost urban space given over to parking lots yield many difficult transportation problems. Autonomous passenger vehicle technology is a promising solution to those problems.

Work by Dickmanns [1] and NavLab [2] at Carnegie Mellon University demonstrated semi-autonomous driving with vision-based nagivation systems in the late 1980s. Since then, many path generation schemes, like arc-line [3], spiral[4] and polynomial spline[5], have been developed for mobile robots. These path generation methods specify only the geometric curve (path), without simultaneously generating the speed plan (trajectory). Trajectory planning was then studied in many race car projects. [6], [7] and [8] used convex optimization routines to generate trajectories that push the race cars to their limits, accounting for complex vehicle dynamics. These offline methods are computationally unaffordable for real-time application.

The 2005 Grand Challenge and the 2007 Urban Challenge made further progress in creating fully autonomous vehicles. For both contests, maps were given, on which the entries used high-accuracy GPS to register the host vehicle. Grand Challenge-winning entry Stanley [9] sampled trajectories in the control space based on the waypoints corridor from the map. Urban Challenge-winning entry Boss [10] spawned short-horizon reactive trajectories by laterally shifting from the lane centerline and picking the fastest collision-free one for execution. The reactive nature of both short-horizon planning methods makes them only applicable for the simplified contest environments.

\footnotetext{
${ }^{1}$ Tianyu $\mathrm{Gu}$ is with the Department of Electrical \& Computer Engineering, Carnegie Mellon University tianyu@ cmu . edu

${ }^{2}$ John M. Dolan is with the Department of Electrical \& Computer Engineering and the Robotics Institute, School of Computer Science, Carnegie Mellon University
}

A deliberative planning quality was later introduced in several lattice-based trajectory sampling methods [11], [12]. Reactiveness was maintained through exhaustive sampling and the computation overhead was significant. [13], [14] reduced the computational overhead by performing two-step (coarse and fine) trajectory planning.

These prior works have demonstrated the technological feasibility of achieving vehicle autonomy. They usually had weighted cost terms as the measurement of optimality. Costs were often manually tuned for maximum performance (speed), rather than systematically adjusted for passenger comfort based on human driving patterns. Only a few works have concerned human driving patterns, for example: [15] investigated the proper speed model that explains a driver's modulation of speed when entering or exiting a curve. [16] attempted to identify abnormal on-road driving behavior in freeway situations by learning a steering-speed mapping. Both works assumes overly simplified models that were not expressive in characterizing general curve negotiation, especially for high-curvature turnings.

\section{SCOPE OF STUDY}

"Human-like" planning is defined as an effort to imitate human driving patterns while staying within the bounds of safe driving. Individuals are likely to have different driving styles; for example, a smooth turning maneuver by an elderly driver may be too slow for young drivers.

In the high-level view of our motion planner (Fig. 1), traffic-free planning generates a reference trajectory considering only road geometry while other traffic and obstacles are ignored. Traffic-based planning produces a spatially and temporally varied reference based on the traffic-free plan to account for other traffic and interfering objects. The tracking trajectory module then generates a dynamically feasible trajectory through model-based evaluation for tracking control.

\begin{tabular}{|c|c|c|c|}
\hline \multicolumn{2}{|c|}{ Reference Planning } & $\begin{array}{c}\text { Controller Interfacing } \\
\text { Traffic-Free } \\
\text { Planning }\end{array}$ \\
\cline { 1 - 3 } & $\rightarrow$ & $\begin{array}{c}\text { Traffic-Based } \\
\text { Planning }\end{array}$ & $\begin{array}{c}\text { Tracking Trajectory } \\
\text { Generation } \\
-\end{array}$ \\
\hline
\end{tabular}

Fig. 1: Planning Structure. On-road navigation uses two planning phases (traffic-free \& traffic-based) and one trajectory generation phase for tracking control. The focus of this paper is on the traffic-free reference planning in bold font.

The focus of this paper is to investigate the appropriate model and the parameter learning methods for urban trafficfree planning, as illustrated by Fig. 2. The rest of this paper is arranged as follows. Section III proposes the expressive 


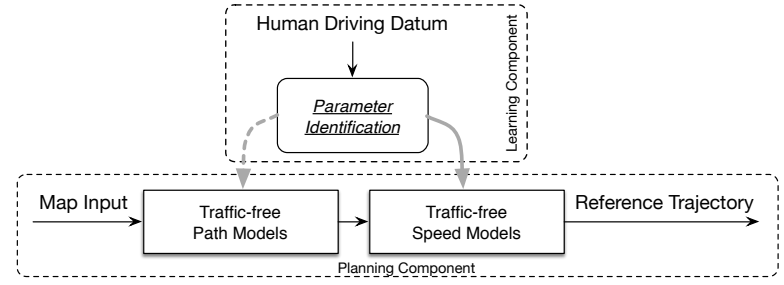

Fig. 2: Overview of human-like traffic-free model learning and planning. It consists of a planning component that generates the human-like traffic-free reference plan, and a learning component that performs parameter identification and statistical interpretation.

trajectory models to capture human driving patterns. Section IV formulates the model identification problems. Sections $\mathrm{V}$ and VI illustrate the results of learning, summarize the contribution and propose future work.

\section{Model Design}

In this section, models are proposed to fit human maneuver data and to be efficiently evaluated for regeneration. To efficiently serve these two purposes, path and speed models are devised and explained independently.

\section{A. Path Model}

The path model makes use of a pre-stored digital map to generate a smooth reference path. Road information is represented by a sequence of map waypoints in a global two-dimensional plane, augmented with lane width as well as speed limits. The map waypoints are usually not distributed uniformly, the interval sometimes spanning from $5 \mathrm{~m}$ to $300 \mathrm{~m}$. To generate a smooth reference path in a sequence of dense and uniformly sampled waypoints, the proposed path model has three steps (Fig. 3).

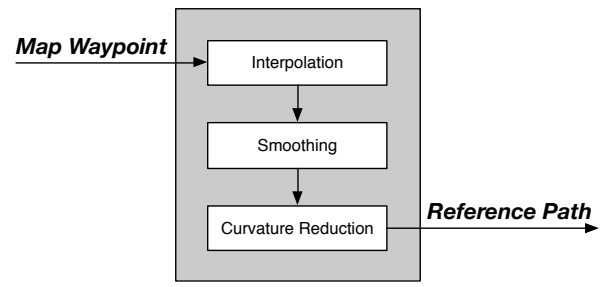

Fig. 3: Path Model. The path model takes three steps to generate a smooth human-like reference path, including interpolation, smoothing and curvature reduction.

The centerline is first generated by connecting 2D map waypoints $(X, Y)$ with interpolation. Neither the $x$ nor the $y$ coordinates of the map waypoints are necessarily monotone. We create a monotonically increasing variable "station" $(s)$ by (linearly) estimating the longitudinal position of each waypoint. Splines interpolate the map waypoints smoothly by separately generating two spline functions $x(s)$ and $y(s)$, which are then evaluated at uniformly sampled stations to represent the centerline in a non-parametric form:

$$
\left\{s_{i},\left[x_{i}, y_{i}\right], \theta_{i}, \kappa_{i}\right\}
$$

where $s_{i},\left[x_{i}, y_{i}\right], \theta_{i}$ and $\kappa_{i}$ respectively represent the $i^{\text {th }}$ longitudinal station, global coordinates, global heading and path curvature. Note that with the polynomial representations, $\theta_{i}$ and $\kappa_{i}$ can be calculated analytically from their definitions.

However, this centerline can easily create high-curvature bumps near waypoints, which causes un-humanlike jerky steering with controllers sensitive to the jerky curvature. Note that experienced drivers do not always stay precisely on the centerline. We use a local smoothing routine to create a smooth result. Two independent smoothings (quadratic least-square fitting) are performed locally to get two smooth polynomial functions $x^{*}(s)$ and $y^{*}(s)$. The size of the smoothing window is chosen to constrain the maximum shift. Polynomial evaluation is conducted similarly as before to obtain a non-parametric form:

$$
\left\{s_{i},\left[x_{i}^{*}, y_{i}^{*}\right], \theta_{i}^{*}, \kappa_{i}^{*}\right\}
$$

Experienced drivers also take advantage of lane width to reduce the maximum curvature during tight turn negotiations. The subsequent optimization for curvature reduction provides visible benefits in such situations. It minimizes the following cumulative term by laterally nudging the points $\left\{p_{i}\right\}$ :

$$
\left\{o_{i}^{*}\right\}=\underset{\left\{o_{i}\right\}}{\operatorname{argmin}} \sum\left|\frac{p_{i}-p_{i-1}}{\left|p_{i}-p_{i-1}\right|}-\frac{p_{i+1}-p_{i}}{\left|p_{i+1}-p_{i}\right|}\right|
$$

where

$$
p_{i}=\left[\begin{array}{c}
x_{i}^{*}-o_{i} \cdot \sin \left(\theta_{i}^{*}\right) \\
y_{i}^{*}+o_{i} \cdot \cos \left(\theta_{i}^{*}\right)
\end{array}\right]
$$

where $o_{i}$ is the lateral nudge distance. Note that this optimization is applied over the region whose absolute value of curvature is above $\rho \cdot \kappa^{\max }$, where $\kappa^{\max }$ is the maximum curvature corresponding to the steering angle limit of the vehicle and $\rho$ is a percentage threshold.

The optimality criterion has a least-square formulation. The Levenberg-Marquardt algorithm is used to numerically solve this optimization problem quickly in a local sense. The overall output of the path model is the reference path.

\section{B. Speed Model}

With the smooth reference path generated, the speed model specifies the speed of the vehicle's motion. Together, they form a reference trajectory. The trajectory determines many dynamic indicators that are important to a passenger's experience of comfort, like lateral \& longitudinal acceleration.

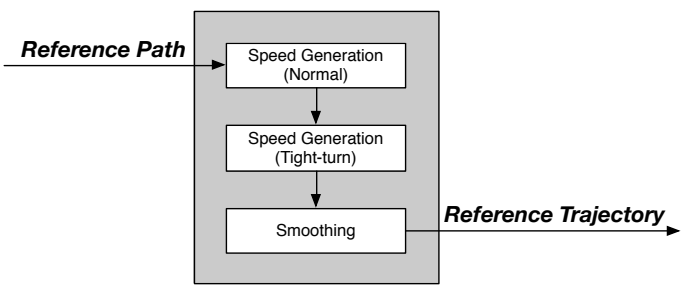

Fig. 4: Speed Model. The speed model takes three steps (normal \& tight-turn speed generation and smoothing) to generate a smooth human-like reference trajectory. 
A three-step speed model is designed to reflect a human driving pattern (Fig. 4). The first step uses the geometry information of the reference path to generate a baseline speed profile for general urban driving cases. For many road segments, speed limits can be obtained from the map. Driving at these speed limits might already yield the optimal speed model, such as in most highway situations. For situations like driving on many curvy urban roads or traversing intersections, however, a valid nominal speed might not exist in the map, so a proper speed profile should be generated in response to the path geometry. We use the normal speed model $M^{\text {normal }}$ :

$$
\left\{v_{i}\right\}=M^{\text {normal }}\left(\left\{\kappa_{i}\right\}, v^{\max }, \mathbf{P}\right)
$$

such that

$$
\begin{aligned}
v_{i} & \leq v^{\max } \\
\kappa_{i} \cdot v_{i}{ }^{2} & \leq a_{\text {lat }} \\
\dot{v}_{i} & \leq a_{l o n} \\
\dot{v}_{i} & \geq d_{l o n}
\end{aligned}
$$

where $\left\{\kappa_{i}\right\}$ is the curvature sequence of the reference path, $v^{\max }$ is the speed limit, and $\mathbf{P}=\left[a_{l a t}, a_{l o n}, d_{l o n}\right]^{T}$ are the tunable parameters that consist of preferred lateral acceleration, longitudinal acceleration and deceleration, respectively.

An iterative numerical algorithm [14] is used to apply several constraint parameters, including speed limit, lateral acceleration and longitudinal acceleration and decelerations. Instead of using the vehicle's extreme values of these constraining terms, human-preferred values are identified and applied. Note that by changing the maximum speed at one particular reference point to zero, we can generate stopping speed profiles.

$M^{\text {normal }}$ restricts the local curvature peak to correspond to a local speed minimum. While sufficient for most urban driving, driving behaviors for tight ${ }^{1}$ turning maneuvers cannot be modeled precisely, since human drivers tend to behave in a cautious manner by decelerating earlier to perform corner negotiation at a lower speed, as shown in Fig. 5. Both drivers' minimum speed is reached prior to the maximum curvature. The absence of this feature could potentially cause passengers' anxiety both when entering and exiting the turns.

To model this driving pattern, several path features are marked first to position the speed profile as shown by the upper plot in Fig. 6, including local peak curvature $\kappa_{\text {peak }}$, the center point $s_{p}$ and the length $l_{p}$ representing the "principal" turning region of a tight turning maneuver, defined by the segment whose curvatures are above $\eta \cdot \kappa^{p e a k}$. A tight-turn specific speed method $M^{\text {tight }}$ is proposed to describe a threephase piecewise-linear process that consists of decelerate-toenter, maintain-low-speed, and accelerate-to-exit, as shown by the lower plot in Fig. 6:

$$
\left\{v_{i}\right\}=M^{\text {tight }}\left(\left\{\kappa_{i}\right\}, \mathbf{Q}\right)
$$

\footnotetext{
${ }^{1}$ A "tight" turning manuver is defined as a turning process whose peak curvature is above certain threshold, 0.07 in our setup.
}
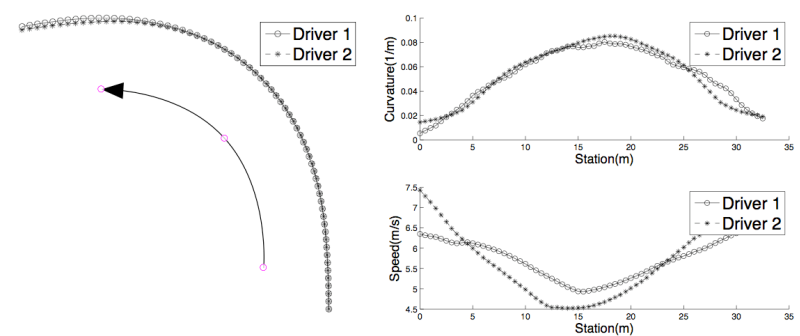

Fig. 5: Tight Turning Maneuver by Human. Human driving data of tight turning maneuvers are collected for two individual drivers on the same road segment. Driver 2 has a more cautious driving style than driver 1 by slowing down earlier and more significantly.

where $\left\{\kappa_{i}\right\}$ is the curvature sequence of the reference path, and $\mathbf{Q}=\left[v_{\text {min }}, s_{\Delta}, l_{v}, \tilde{a}_{l o n}, \tilde{d}_{l o n}\right]^{T}$ defines the shape of the speed profile. Another parameter $s_{\Delta}=s_{p}-s_{v}$ defines the longitudinal distance from the centerpoint of the "principal" turning region to the local speed minimum point.

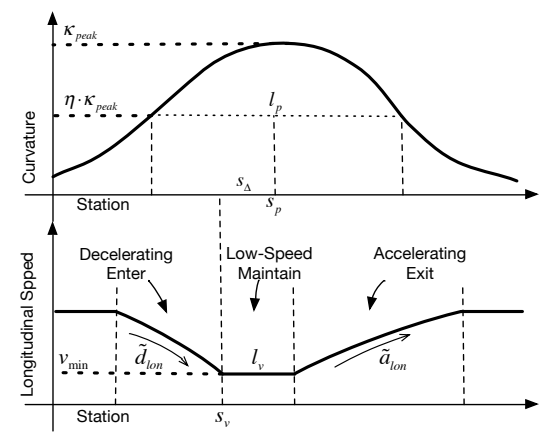

Fig. 6: Tight Turn Speed Model. Plot above illustrates the path features on curvature plot. Plot below shows the threephase speed model for a tight-turning maneuver.

Note that $M^{\text {tight }}$, as well as $M^{\text {normal }}$, potentially yields speed profiles with non-smooth (huge longitudinal jerk value) transition points connecting linear segments. In order to improve the speed smoothness, the third step constrains the numerically interpreted jerk iteratively until the maximum jerk value is below a certain threshold.

$$
|\ddot{v}| \leq j_{l o n}
$$

\section{Model FitTing}

The proposed models have multiple parameters to be fitted from the human driving data. The path model performs smoothing and optimization with respect to two curvaturerelated criterias, i.e. smoothness and peak value. We assume that a natural human driving pattern is to achieve optimalities in these two aspects, in which case, path model fitting (and subsequent learning) becomes unnecessary. The focus of this section is to identify the parameters of the speed models $M^{\text {normal }}$ and $M^{\text {tight }}$.

The parameters $\mathbf{P}$ of $M^{\text {normal }}$ are fitted by performing an optimization that minimizes the least-square error for model 
$M^{\text {normal }}$ and human driving data:

$$
\hat{\mathbf{P}}=\underset{\mathbf{P}}{\operatorname{argmin}}\left\|\left\{v_{i}^{\text {human }}\right\}-M^{\text {normal }}\left(\left\{\kappa_{i}\right\}, v^{\text {limit }}, \mathbf{P}\right)\right\|
$$

where $\left\{v_{i}^{\text {human }}\right\}$ represents the human driving speed data for normal urban curves. Note that the parameter $a_{l a t}$ in $\mathbf{P}$ can be determined by other methods, as will be shown in the following section. Only the two remaining parameters in $\mathbf{P}$ will be identified with equation (5).

The parameters $\mathbf{Q}$ of model $M^{\text {tight }}$ are fitted with an optimization via a similar least-square error formulation:

$$
\hat{\mathbf{Q}}=\underset{\mathbf{Q}}{\operatorname{argmin}}\left\|\left\{v_{i}^{\text {human }}\right\}-M^{\text {tight }}\left(\left\{\kappa_{i}\right\}, \mathbf{Q}\right)\right\|
$$

where $\left\{v_{i}^{\text {human }}\right\}$ represents the human driving speed data on tight turns. Note that the parameter $v_{\text {min }}$ in $\mathbf{Q}$ can be obtained simply by scanning the human driving data to fit. Only the four remaining parameters in $\mathbf{Q}$ will be identified with equation (6).

In Fig. 7, an example turning maneuver is fitted with the appropriate optimization routine above, in which the speed model shows good adaptability to the human driving pattern.
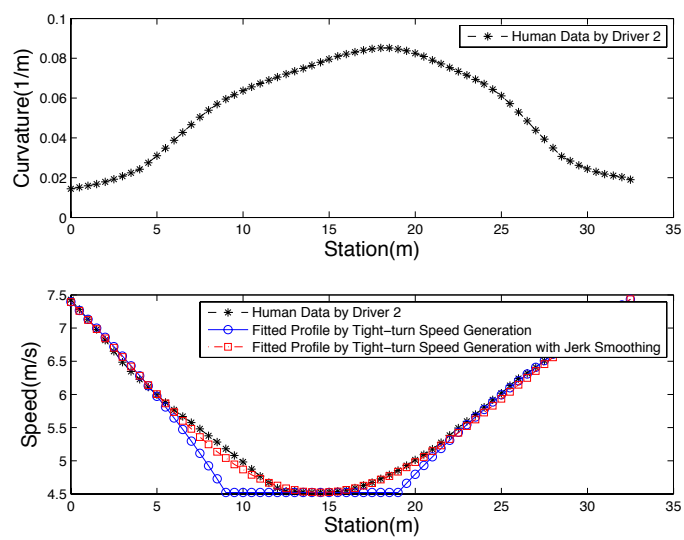

Fig. 7: Speed Model Fitting Example. The black dashed star curves represent the human driving. The blue solid circle curve is the speed profile fitting speed model $M^{\text {tight }}$. The red dashed square curve is after further applying jerk smoothing.

\section{EXPERIMENTS AND RESULTS}

Two experiments were designed to support the claims of this paper. The first explains parameter learning results of the proposed speed models. The second evaluates the learned model by comparing the plan with real human driving data.

\section{A. Parameter Learning}

1) Data collection: In order to obtain human driving data, we set up a differential GPS-based localization system mounted at the center of the rear axle. An experienced human driver was required to drive in a comfortable manner in three distinct scenarios including highway $\left(S_{1}\right)$, urban roads $\left(S_{2}\right)$ and parking lots with multiple tight turns $\left(S_{3}\right)$, as illustrated in Fig. 8. The high-accuracy output data consisting of po-

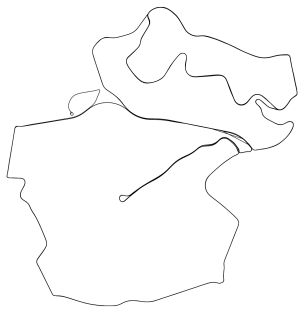

(b)

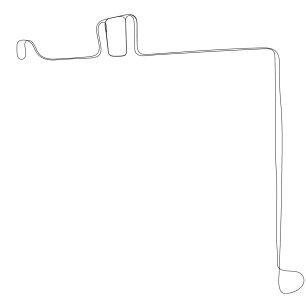

(c)
Fig. 8: Three experiment scenarios. (a) $S_{1}$ : Highway $\mathrm{I} 376$ and 179 connecting $S_{2}$ and $S_{3}$. (b) $S_{2}$ : Urban driveways near Schenley Park, Overlook Dr, Pittsburgh, PA, USA. (c) $S_{3}$ : parking lots near Ernie Mashuda Dr, Cranberry, PA, USA.

sition, heading and path curvature, as well as longitudinal speed and acceleration were recorded:

$$
\left\{\left[x_{i}^{\text {human }}, y_{i}^{\text {human }}, \theta_{i}^{\text {human }}, \kappa_{i}^{\text {human }}, v_{i}^{\text {human }}, a_{i}^{\text {human }}\right]\right\}
$$

2) Learning: Learning was performed to distill and discover important characteristics of human driving pattern. Due to the variations in human behavior, two types of statistical regression approaches were performed after identifying the model parameters at the multiple curve negotiation locations.

Three parameters of the speed model $M^{\text {normal }}$ must be identified. We use an asterisk to represent a statistically learned parameter, e.g. $\mathbf{P}^{*}=\left[a_{\text {lat }}^{*}, a_{\text {lon }}^{*}, d_{l o n}^{*}\right]^{T}$. The preferred lateral acceleration $a_{\text {lat }}^{*}$ is first learned from the data by creating a state-action mapping, where the "state" is the curvature of the reference path and the "action" is the value of $a_{\text {lat }}$. Scattering $\left\{\left[\kappa_{i}^{\text {human }}, v_{i}^{\text {human }}\right]\right\}$ data points on the curvature-speed plane (scattered grey circles in Fig. 12) makes a clear capping curve visible to characterize the preferred maximum speed at a given path curvature. However, it is unknown what the analytical expression should be. [17] proposed a computational search method (Fig. 9) that performs symbolic regression on the experimental data, simultaneously fitting both the symbolic form ${ }^{2}$ and its parameters.

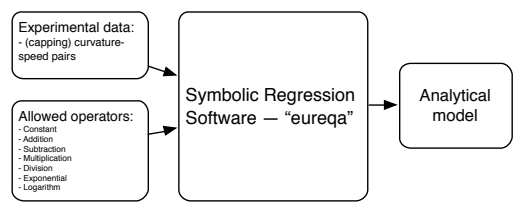

Fig. 9: Symbolic Regression. The symbolic regression routine takes the allowed operations to distill an analytical model that best explains the experimental data.

In practice, we first find an analytical expression $v_{\max }^{*}(\kappa)$ that describes the capping curves based on the data points collected from all $S_{1}, S_{2}$ and $S_{3}$ scenarios with the analytical

\footnotetext{
${ }^{2}$ By restricting the allowed operators, we obtain certain control over the complexity of this symbolic form.
} 


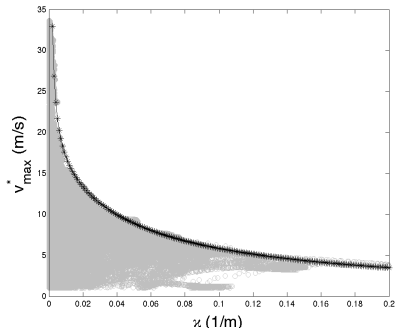

(a)

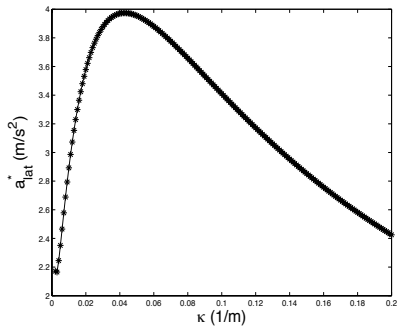

(b)
Fig. 10: Symbolic regression result from human driving data. (a) shows the scattered $\left\{\left[\kappa_{i}^{\text {human }}, v_{i}^{\text {human }}\right]\right\}$ human driving datum and the plot of analytical capping curve. (b) shows the learned curvature $\rightarrow$ lateral acceleration mapping.

expression (Fig. 10a):

$$
v_{\text {max }}^{*}=\frac{0.0348}{|\kappa|}+\frac{0.832}{0.0515+|\kappa|}
$$

The mapping of $a_{\text {lat }}{ }^{*}$ can further be calculated with the following equation, as shown in Fig. 10b:

$$
\begin{aligned}
a_{\text {lat }}{ }^{*}(\kappa) & =\kappa \cdot v_{\text {max }}^{*}{ }^{2} \\
& =\frac{0.6922|\kappa|}{(|\kappa|+0.0515)^{2}}+\frac{0.05791}{|\kappa|+0.0515}+\frac{0.0012}{|\kappa|}
\end{aligned}
$$

For $a_{l o n}^{*}$ and $d_{l o n}^{*}$, multiple parameter values are obtained by model fitting of different (traffic-free) curve negotiation maneuvers from scenario $S_{2}$. A relation can be discovered by linear regression:

$$
\begin{aligned}
& a_{\text {lon }}^{*}\left(\kappa^{\text {peak }}\right)=0.2453+6.7456 \cdot\left|\kappa^{\text {peak }}\right| \\
& d_{\text {lon }}^{*}\left(\kappa^{\text {peak }}\right)=0.1366+10.5464 \cdot\left|\kappa^{\text {peak }}\right|
\end{aligned}
$$

As for the speed model $M^{\text {tight }}$, five parameters must be identified, i.e., $\mathbf{Q}^{*}=\left[v_{m i n}^{*}, s_{\Delta}^{*}, l_{v}^{*}, \tilde{a}_{l o n}^{*}, \tilde{d}_{l o n}^{*}\right]^{T}$. Tight turning maneuvers from scenario $S_{3}$ are used to fit the multiple recorded turns. Similarly, linear regression learns a statistical mapping of the parameters we are interested in.

$$
\begin{aligned}
v_{\text {min }}^{*}\left(\kappa^{\text {peak }}\right) & =7.5534-28.4011 \cdot\left|\kappa^{\text {peak }}\right| \\
s_{\Delta}^{*}\left(\kappa^{\text {peak }}\right) & =1.6591+50.0945 \cdot\left|\kappa^{\text {peak }}\right| \\
l_{v}^{*}\left(l_{p}\right) & =1.1873+0.4517 \cdot l_{p} \\
\tilde{a}_{\text {lon }}^{*}\left(\kappa^{\text {peak }}\right) & =1.3784-2.2145 \cdot\left|\kappa^{\text {peak }}\right| \\
\tilde{d}_{\text {lon }}^{*}\left(\kappa^{\text {peak }}\right) & =1.3746+1.8192 \cdot\left|\kappa^{\text {peak }}\right|
\end{aligned}
$$

3) Discussion: The symbolic regression discovered a new relation between $a_{l a t}^{*}$ and the path geometry, which potentially can be adapted to other human driviers with its three coefficients. The other parameters showed both strong and weak correlation. The closer their determination coefficients $\left(R^{2}\right)$ are to 1 , the stronger linearity can be inferred. Based on Table I and the scattering plots of each parameter (Fig. 11), we discover some human driving patterns that can be explained in a statistical sense:

1) $v_{\min }^{*}$ demonstrates the strongest linearity. It shows that the tighter the maximimum curvature of a negotiation,
TABLE I: Determination coefficients of linear models

\begin{tabular}{c|c||c|c|c|c|c}
\hline$R_{a_{\text {lon }}^{*}}^{2}$ & $R_{d_{l o n}^{*}}^{2}$ & $R_{\tilde{a}_{l o n}^{*}}^{2}$ & $R_{\tilde{d}_{l o n}^{*}}^{2}$ & $R_{v_{\text {min }}^{*}}^{2}$ & $R_{s_{\Delta}^{*}}^{2}$ & $R_{l_{v}^{*}}^{2}$ \\
\hline 0.34 & 0.29 & 0.02 & 0.01 & 0.68 & 0.08 & 0.58 \\
\hline
\end{tabular}

the lower the maintaining speed $v_{\min }$ will be. The necessity of speed model $M^{\text {tight }}$ is also demonstrated, since the learned maintaining speed $v_{m i n}^{*}$ is significantly lower than the preferred maximum speed $v_{\max }^{*}$ of speed model $M^{\text {normal }}$.

2) $s_{\Delta}^{*}$ demonstrates a weak linear correlation with $\kappa_{\text {peak }}$ overall. This relation can be interpreted as meaning that as the maximum curvature of a maneuver increases, the driver tends to behave more cautiously be slowing down earlier. The linearity is stronger when $\kappa_{\text {peak }}$ is smaller and vice versa, which may also imply that the human driver tends to behave more unpredictably during high-curvature negotations.

3) The mapping between $l_{p}$ and $l_{v}$ shows the second strongest linearity. This has an intuitive explanation: the longer the "principal" turning process lasts, the longer the low-speed-maintaining process will be.

4) $a_{l o n}^{*}$ and $d_{l o n}^{*}$ from model $M^{\text {normal }}$ clearly show stronger linearity than $\tilde{a}_{l o n}^{*}$ and $\tilde{d}_{l o n}^{*}$ from model $M^{\text {tight }}$, which also reflects that the human driver tends to behave more unpredictably during high-curvature negotations. But overall, they all have rather low determination coefficient values, confirming the argument made in [16] that the longitudinal accelerations have a great variance depending on hard-to-access variables like driver mood.

\section{B. Model Evaluation}

1) Evaluation: The learned models $M^{\text {normal }}{ }^{*}$ and $M^{\text {tight* }}$ are fully specified by parameters $\mathbf{P}^{*}$ and $\mathbf{Q}^{*}$, respectively. To evaluate the quality of the models, speed plans are generated and compared with two human driving processes.

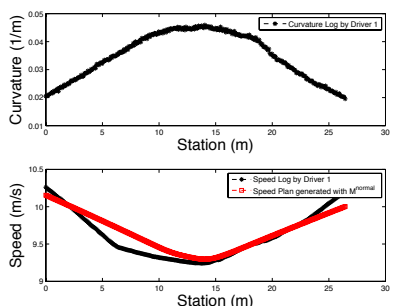

(a)

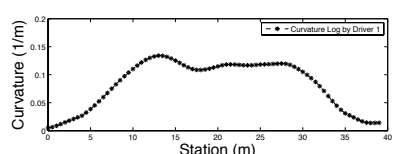

Station (m)

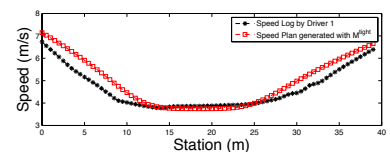

(b)
Fig. 12: Learned speed model evaluation result. (a) evaluate the planning output of $M^{\text {normal }}{ }^{*}$ on a mild turn. (b) evaluate the planning output of $M^{\text {tight }}{ }^{*}$ on a tight turn.

2) Discussion: From the evaluation results of both $M^{\text {normal }}{ }^{*}$ and $M^{\text {tight }}{ }^{*}$ speed models, it is illustrated that the plan generated can rarely match perfectly with any one-time human data. But we are able to generate traffic-free reference 


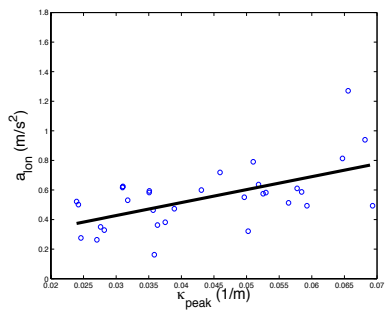

(a)

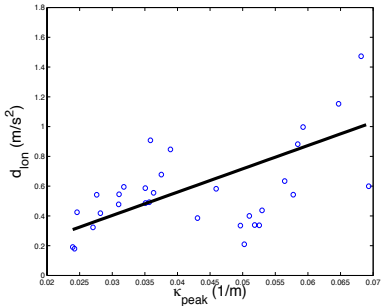

(b)

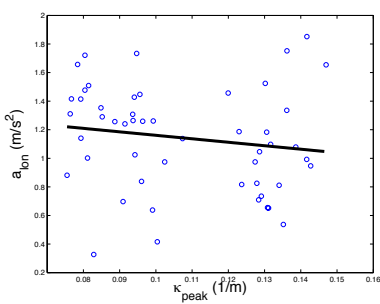

(c)

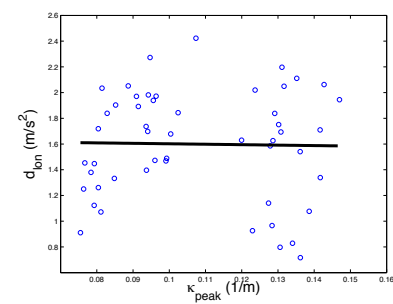

(d)

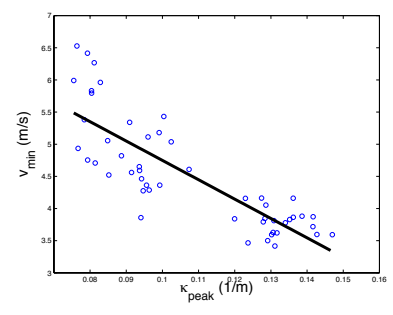

(e)

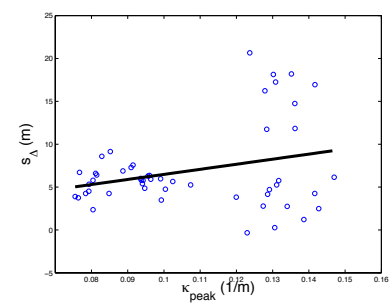

(f)

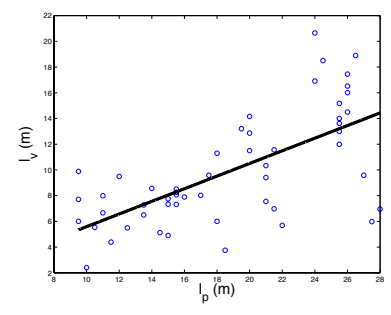

$(\mathrm{g})$

Fig. 11: Scattering plot of the speed model $M^{\text {normal }}$ and $M^{\text {tight }}$ parameters. From subfigure (a) to (g), blue circles are the scattered parameter values after model fitting. Black lines are the results after linear regression.

plans that capture the key human features (in the statistical sense) of both mild and tight turning maneuvers. Moreover, using these learned models guarantees the smoothness and predictability of planning results.

\section{CONCLUSION}

The general on-road planning is decoupled into trafficfree and traffic-based planning procedures. In this paper, efforts are made toward human-like traffic-free planning in order to improve the user experience of autonomous driving. Expressive and tunable trajectory models suitable for mimicking human traffic-free driving are proposed. Parameter identifications are formulated and solved as least-square optimization problems. The parameters are then generalized statistically with regression methods in order to characterize the driving patterns. Meanwhile, the regression characteristics also reflect the sensibility of each parameter to the unpredictability of human driving. Finally, the learned model is evaluated on a new data set and is able to generate smooth planning result that captures the user's driving pattern.

One limitation of this work is that the reference speed model is completely built on the geometric nature of the reference path, while other factors affecting traffic-free human driving are ignored, like weather/road condition. An immediate next step is to apply the learned model to the planning system of a real autonomous car. Also, more human driving data by different people must be collected and analyzed in order to generate user-specific model parameters. Moreover, human-like traffic-based planning models and their parameter learning methods should be studied to handle dynamic environments.

\section{REFERENCES}

[1] E. D. Dickmanns et al., "The seeing passenger car 'VaMoRs-P'," in Proceedings of the IEEE Intelligent Vehicles '94 Symposium, pp. 6873, 1994.
[2] C. Thorpe et al., "Vision and navigation for the Carnegie-Mellon Navlab," IEEE Transactions on Pattern Analysis and Machine Intelligence, vol. 10, no. 3, pp. 362-373, 1988.

[3] Y. Kanayama and B. I. Hartman, Smooth local path planning for autonomous vehicles. Springer, 1990.

[4] A. Kelly and B. Nagy, "Reactive nonholonomic trajectory generation via parametric optimal control," The International Journal of Robotics Research, vol. 22, no. 7-8, pp. 583-601, 2003.

[5] A. Piazzi and C. Guarino Lo Bianco, "Quintic $g_{i} \sup _{i} 2_{i} /$ sup $_{i}$-splines for trajectory planning of autonomous vehicles," in Proceedings of the IEEE Intelligent Vehicles Symposium (IV), pp. 198-203, 2000.

[6] E. Velenis and P. Tsiotras, "Minimum time vs maximum exit velocity path optimization during cornering," in IEEE international symposium on industrial electronics, pp. 355-360, 2005.

[7] E. Velenis and P. Tsiotras, "Optimal velocity profile generation for given acceleration limits: Theoretical analysis," in Proceedings of the IEEE American Control Conference, pp. 1478-1483, 2005.

[8] J. Funke et al., "Up to the limits: Autonomous audi tts," in IEEE Intelligent Vehicles Symposium (IV), pp. 541-547, 2012.

[9] S. Thrun et al., "Stanley: The robot that won the darpa grand challenge," Journal of field Robotics, vol. 23, no. 9, pp. 661-692, 2006.

[10] C. Urmson et al., "Autonomous driving in urban environments: Boss and the urban challenge," Journal of Field Robotics, vol. 25, no. 8, pp. 425-466, 2008.

[11] M. Werling et al., "Optimal trajectories for time-critical street scenarios using discretized terminal manifolds," International Journal of Robotics Research, vol. 31, pp. 346-359, Mar. 2012.

[12] M. McNaughton, "Parallel algorithms for real-time motion planning," PhD Thesis, Carnegie Mellon University, 2011.

[13] T. Gu and J. M. Dolan, "On-road motion planning for autonomous vehicles," in Intelligent Robotics and Applications, pp. 588-597, Springer Berlin Heidelberg, 2012.

[14] T. Gu et al., "Focused trajectory planning for autonomous on-road driving," in IEEE Intelligent Vehicles Symposium (IV), pp. 547-552, 2013.

[15] G. Reymond et al., "Role of lateral acceleration in curve driving: Driver model and experiments on a real vehicle and a driving simulator," Human Factors: The Journal of the Human Factors and Ergonomics Society, vol. 43, no. 3, pp. 483-495, 2001.

[16] I. Markelic et al., "The driving school system: Learning basic driving skills from a teacher in a real car," IEEE Transactions on Intelligent Transportation Systems, vol. 12, no. 4, pp. 1135-1146, 2011.

[17] M. Schmidt and H. Lipson, "Distilling free-form natural laws from experimental data," science, 2009. 